\title{
Resection of a Retroperitoneal Bronchogenic Cyst: Laparoscopic Approach
}

\author{
Agustin Dietrich, Martin de Santibañes, Fanny Rodriguez Santos, Juan Santino, Agustin Cristiano, \\ Rodrigo Sanchez Clariá \\ Department of General Surgery, Hospital Italiano de Buenos Aires, Argentina (Drs. Dietrich, Santos). \\ Department of HPB Surgery, Hospital Italiano de Buenos Aires, Argentina (Drs. de Santibañes, Cristiano, Sanchez Clariá). \\ Department of Pathology, Hospital Italiano de Buenos Aires, Argentina (Dr. Santino).
}

\begin{abstract}
Bronchogenic cysts are rare, benign, congenital anomalies of the primitive foregut encountered in the posterior mediastinum. Retroperitoneal location is uncommon, with only a few cases reported in the literature.

We present a 46-year-old man who was examined for a complaint of nonspecific chronic abdominal pain. An abdominal computed tomography scan and magnetic resonance imaging scan were performed and revealed a mass in the peripancreatic region in relation to the left adrenal gland. Because a definitive diagnosis was uncertain, the patient underwent a laparoscopic resection. The pathology showed a cystic lesion consistent with retroperitoneal bronchogenic cyst. The patient had an uneventful postoperative recovery.
\end{abstract}

Surgical resection of these lesions is mandatory to arrive at a differential diagnosis with other retroperitoneal lesions. The laparoscopic approach should be performed by experienced surgeons.

Key Words: Bronchogenic cysts, Retroperitoneal mass, Laparoscopic resection.

Citation Dietrich A, de Santibañes M, Rodriguez Santos F, Santino J, Cristiano A, Sanchez Clariá R. Resection of a retroperitoneal bronchogenic cyst: laparoscopic approach. CRSLS e2014.00126. DOI: 10.4293/CRSLS.2014.00126.

Copyright (C) 2014 SLS This is an open-access article distributed under the terms of the Creative Commons Attribution-Noncommercial-ShareAlike 3.0 Unported license, which permits unrestricted noncommercial use, distribution, and reproduction in any medium, provided the original author and source are credited.

Address correspondence to: Agustin Dietrich, M.D., General Surgical Service, Hospital Italiano de Buenos Aires, Juan D. Perón 4190, C1181ACH. Buenos Aires,

Argentina, E-mail address: agustin.dietrich@hospitalitaliano.org.ar

\section{INTRODUCTION}

Bronchogenic cysts are rare congenital anomalies of the primitive foregut that occur during early embryogenesis. They usually develop in the posterior mediastinum, most commonly along the tracheobronchial tree. ${ }^{1}$ Although retroperitoneal location has been previously described, it is extremely rare. ${ }^{2-4}$ Patients usually remain asymptomatic and most of these tumors are discovered incidentally.5,6

We present the case of a patient with a retroperitoneal cyst who underwent a laparoscopic resection with a postoperative histopathologic diagnosis of retroperitoneal bronchogenic cyst.

\section{CASE REPORT}

A 46-year-old man with an unremarkable previous medical history underwent examination for nonspecific chronic abdominal pain. Multidetector computed tomography
(MDCT) of the abdomen and pelvis showed a 35-mm hypodense well-circumscribed lesion in close relation to the body of the pancreas and the left adrenal gland, without any other significant findings. The study was completed with magnetic resonance imaging (MRI) scan, which showed a high-intensity lesion on T2-weighted images that confirmed the probable cystic origin of the lesion (Figure 1).

The clinical case was discussed in a multidisciplinary scenario. Surgical exploration was decided on the basis of observing this retroperitoneal cyst of unknown origin.

The patient was placed in the lithotomy position with the table tilted head-up. Three trocars were placed: a $12-\mathrm{mm}$ trocar in the midline of the abdominal wall, a $12-\mathrm{mm}$ placed laterally, and an epigastric trocar.

Surgery began with complete exposure of the body and tail of the pancreas through an opening in the gastrocolic liga- 


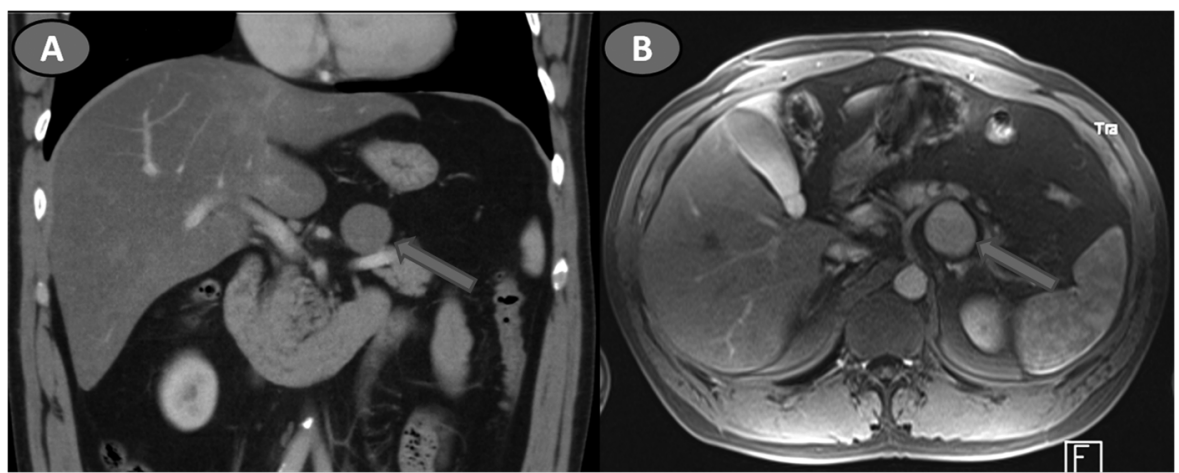

Figure 1. MDCT and abdominal MRI images showing the dense cystic mass in the left suprarenal region adjacent to the pancreas (blue arrow).

ment. On the upper edge of the pancreas, the cystic lesion was visualized (Figure 2). The tumor was mobilized, thus releasing multiple retroperitoneal adhesions. The cyst was then resected, with care taken to not damage the pancreatic body and the celiac trunk. The resected tumor was placed in a plastic bag and removed from the abdomen by extending the port orifice. Because of the close relationship with the pancreas surface, an abdominal drain was placed.

The patient had an uneventful postoperative course and was discharged on postoperative day 3 .

The histopathology examination showed a pseudostratified, ciliated, columnar epithelium (respiratory-type epithelium); fibrous connective tissue; and focal seromucous glands. The entire cyst was studied but no cartilage was found. The final diagnosis was a retroperitoneal bronchogenic cyst (Figure 3).

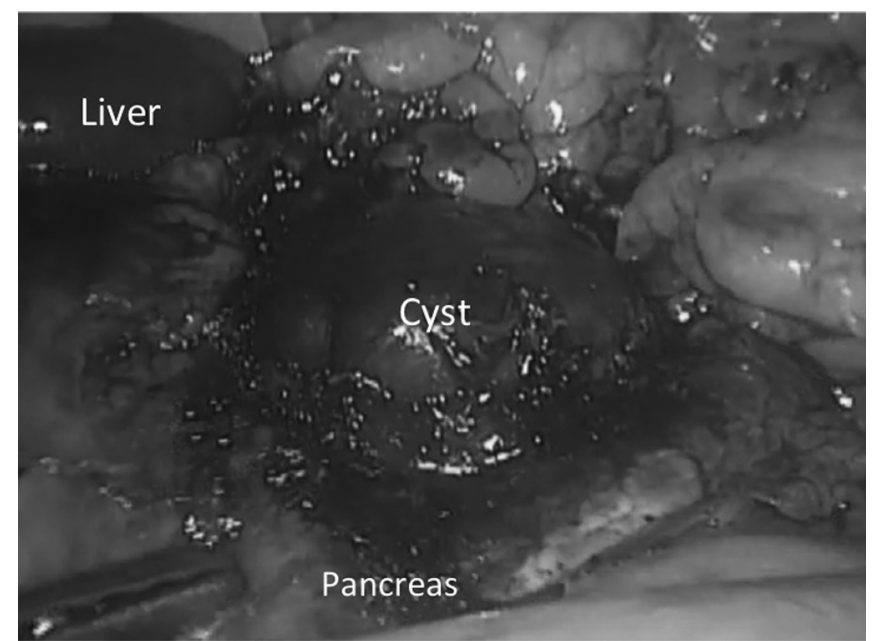

Figure 2. Intraoperative snapshot that shows the cystic lesion in close relation to the pancreas.

\section{DISCUSSION}

The first report of a retroperitoneal bronchogenic cyst was described in 1953 by Miller et al. ${ }^{7}$ These tumors are commonly located in the thoracic cavity and retroperitoneal localization is extremely rare. The peripancreatic region ${ }^{8}$ and the left adrenal gland ${ }^{9}$ are the most common localizations of the bronchogenic cysts placed in the retroperitoneum.

The pathogenesis of retroperitoneal bronchogenic cysts is still unknown. Sumiyoshi et a ${ }^{10}$ described a lack in closure of the pericardioperitoneal channel during embryological development, causing the migration of elements of the tracheobronchial tree to the abdominal cavity. Another theory suggested that retroperitoneal bronchogenic cysts could be regarded as abnormally differentiated foregut duplication cysts, explaining their close relationship with organs derived from the primitive foregut, such as the pancreas or gallbladder. ${ }^{11}$

Bronchogenic cysts are commonly asymptomatic and their diagnosis is made incidentally. ${ }^{9}$ Our patient had nonspecific back pain without other symptoms. It has been suggested that these tumors can grow, bleed, or compress adjacent structures, thereby becoming symptomatic. ${ }^{12}$ Infections and cystic perforations have been also described. ${ }^{13}$

Advanced radiological images such as MRI and MDCT have become fundamental to achieve etiological diagnosis of these lesions. In our case, we performed MDCT, which showed a hypo-intense image compatible with a retroperitoneal solid mass. However, the MRI scan revealed a cystic lesion with the presence of highprotein fluid. It has been previously described that in MDCT, bronchogenic cysts can manifest as hypo-intense images without enhancement. Nevertheless, the presence of proteinaceous secretions inside the cyst can lead to misdiagnosis with MDCT. ${ }^{14}$ 


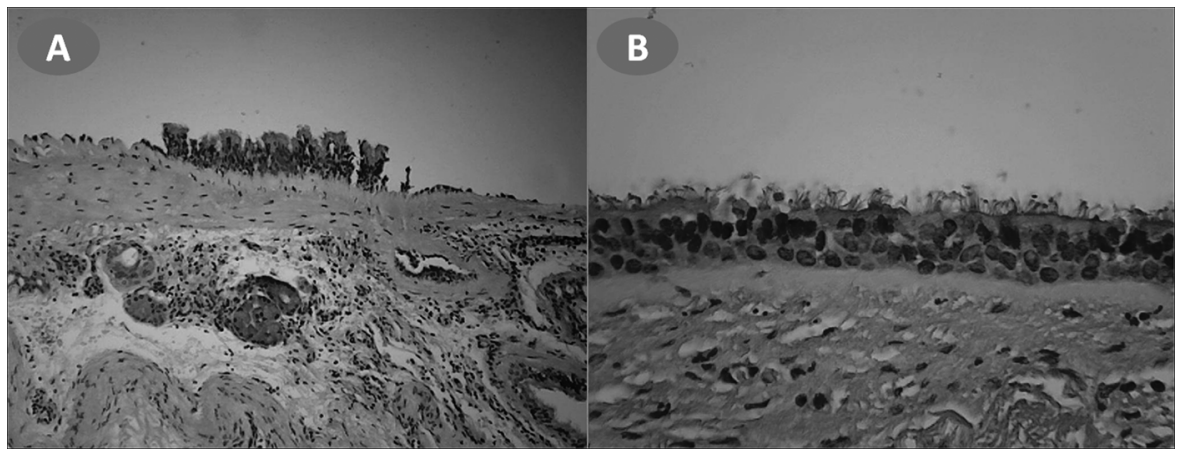

Figure 3. A, The cyst lining composed of respiratory-type epithelium and underlying lamina propia (connective tissue) containing mucous glands (hematoxylin and eosin staining, original magnification $\times 200$ ). B, Ciliated columnar epithelium (hematoxylin and eosin staining; original magnification $\times 400$ ).

Bronchogenic cysts should be differentiated from other retroperitoneal lesions like cystic lymphangioma, cystic teratoma, cystic mesothelioma, and pseudomixoma retroperitonei, or from non-neoplastic lesions such as pancreatic pseudocyst, lymphocele, and hematoma. ${ }^{15}$ Despite the progress in imaging modalities, surgical resection is mandatory to achieve a definitive diagnosis. ${ }^{13,16}$

The benefits of the laparoscopic approach have been widely described to treat retroperitoneal cyst with low morbidity and excellent outcomes. However, this technique should be performed by experienced surgeons because of its complex location. ${ }^{8,17}$

\section{References:}

1. Cuypers P, de Leyn P, Cappelle L, Verougstraete L, Demedts M, Deneffe G. Bronchogenic cysts: a review of 20 cases. Eur J Cardiothorac Surg. 1996;10(6):393-396.

2. Menke H, Röher HD, Gabbert H, Schweden F. Bronchogenic cyst: a rare cause of a retroperitoneal mass. Eur J Surg. 1997; 163(4):311-314.

3. Ingu A, Watanabe A, Ichimiya Y, et al. Retroperitoneal bronchogenic cyst: a case report. Chest. 2002;121:1357-1359.

4. Haddadin WJ, Reid R, Jindal RM. A retroperitoneal bronchogenic cyst: a rare cause of a mass in the adrenal region. J Clin Pathol. 2001;54:801-802.

5. Siegelman ES, Birnbaum BA, Rosato EF. Bronchogenic cyst appearing as a retroperitoneal mass. AJR Am J Roentgenol. 1998; 171:527-528.

6. Murakami R, Machida M, Kobayashi Y, Ogura J, Ichikawa T, Kumazaki T. Retroperitoneal bronchogeniccyst: CT and MR imaging. Abdom Imaging. 2000;25:444-447.

7. Miller RF, Graub M, Pashuck ET. Bronchogenic cysts; anomalies resulting from maldevelopment of the primitive foregut and midgut. Am J Roentgenol Radium Ther Nucl Med. 1953;70:771785 .

8. Wang S-E, Tsai Y-F, Su C-H, et al. Retroperitoneal bronchogenic cyst mimicking pancreatic cystic lesion. J Chinese Med Assoc. 2006;69(11):538-542.

9. Chung JM, Jung MJ, Lee W, Choi S. Retroperitoneal bronchogenic cyst presenting as adrenal tumor in adult successfully treated with retroperitoneal laparoscopic surgery. Urology. 2009; 73:442.e13-442.e15.

10. Sumiyoshi K, Shimizu S, Enjoji M, et al. Bronchogenic cyst in the abdomen. Virchows Arch A Pathol Anat Histopathol. 1985; 408:93-98.

11. El Youssef R, Fleseriu M, Sheppard BC. Adrenal and pancreatic presentation of subdiaphragmatic retroperitoneal bronchogenic cysts. Arch Surg. 2010;145:302-304.

12. Govaerts K, Van Eyken P, Verswijvel G, Van der Speeten K. A bronchogenic cyst, presenting as a retroperitoneal cystic mass. Rare Tumors. 2012;4(1):e13.

13. Coselli MP, de Ipolyi P, Bloss RS, et al. Bronchogenic cysts above and below the diaphragm: report of eight cases. Ann Thorac Surg. 1987;44:491-494.

14. Murakami R, Machida M, Kobayashi Y, et al. Retroperitoneal bronchogenic cyst: CT and MR imaging. Abdom Imaging. 2000; 25:444-447.

15. Yang DM, Jung DH, Kim H, et al. Retroperitoneal cystic masses: CT, clinical, and pathologic findings and literature review. Radiographics. 2004;24(5):1353-1365.

16. Runge T, Blank A, Schäfer SC, Candinas D, Gloor B, Angst E. A retroperitoneal bronchogenic cyst mimicking a pancreatic or adrenal mass. Case Rep Gastroenterol. 2013;7(3):428-432.

17. Diaz Nieto R, Naranjo Torres A, Gomez Alvarez M, et al. Intraabdominal bronchogenic cyst. J Gastrointest Surg. 2010;14: $756-758$. 